\title{
Compensatory Postural Adjustments in an Oculus Virtual Reality Environment and the Risk of Falling in Alzheimer's Disease
}

\author{
Miguel F. Gago ${ }^{a, c, d}$ Darya Yelshyna ${ }^{b}$ Estela Bicho $^{b}$ Hélder David Silva ${ }^{b}$ \\ Luís Rochab Maria Lurdes Rodrigues $^{\mathrm{a}}$ Nuno Sousac, ${ }^{\mathrm{d}}$ \\ ${ }^{a}$ Neurology Department, Hospital da Senhora da Oliveira, EPE, Guimarães, ${ }^{b}$ Centro \\ ALGORITMI, Department of Industrial Electronics, School of Engineering, and ' Life and \\ Health Sciences Research Institute (ICVS), School of Health Sciences, University of Minho, \\ Braga, and dICVS-3Bs PT Government Associate Laboratory, Braga/Guimarães, Portugal
}

Key Words

Alzheimer's disease · Falls - Compensatory postural adjustments - Oculus virtual reality ·

Time-frequency distribution · Inertia measurement units

\section{Abstract}

Background/Aims: Alzheimer's disease (AD) patients have an impaired ability to quickly reweight central sensory dependence in response to unexpected body perturbations. Herein, we aim to study provoked compensatory postural adjustments (CPAs) in a conflicting sensory paradigm with unpredictable visual displacements using virtual reality goggles. Methods: We used kinematic time-frequency analyses of two frequency bands: a low-frequency band (LB; $0.3-1.5 \mathrm{~Hz}$; mechanical strategy) and a high-frequency band (HB; $1.5-3.5 \mathrm{~Hz}$; cognitive strategy). We enrolled 19 healthy subjects (controls) and 21 AD patients, divided according to their previous history of falls. Results: The AD faller group presented higher-power LB $C P A s$, reflecting their worse inherent postural stability. The AD patients had a time lag in their HB CPA reaction. Conclusion: The slower reaction by CPA in AD may be a reflection of different cognitive resources including body schema self-perception, visual motion, depth perception, or a different state of fear and/or anxiety.

\section{Introduction}

Around $30 \%$ of people aged more than 65 years living in the community and more than $50 \%$ of those living in residential care facilities or nursing homes fall every year, and about half of those who fall do so repeatedly [1]. With the growing elderly population, the number 
Gago et al.: Compensatory Postural Adjustments in an Oculus Virtual Reality

Environment and the Risk of Falling in Alzheimer's Disease

of falls in this group has also increased [2]. Postural stability degrades with aging and is a factor for the occurrence of falls, especially in neurodegenerative diseases such as Alzheimer's disease (AD). These balance deficits are characterized by excessive and uncontrolled sway [3]. AD is the major cause of dementia in the geriatric population in the USA and Western Europe, but it is also associated with posture and gait disturbances [4, 5]. In fact, falls are more frequent and have more serious traumatic consequences, including hip fracture, in individuals with $\mathrm{AD}$ than in nondemented elderly people [6]. However, the underlying mechanisms contributing to falls in AD patients are still not clearly understood.

Anticipatory postural adjustments (APAs) and compensatory postural adjustments (CPAs) are the two main mechanisms used by the CNS in order to deal with body perturbations that may either be internally or externally generated [7]. When postural perturbation is unpredictable, postural muscles are activated to restore stability after the moment of perturbation. These later responses (CPAs) are triggered by sensory feedback signals and help in dealing with the actual effects of a perturbation [8, 9]. While APAs are observed only in the case of predictable perturbations, CPAs are seen during both predictable (following APAs) and unpredictable perturbations, which often are of a larger magnitude [10]. The absence or degradation of any type of sensory input or higher cortical control may affect balance performance, that is CPAs [11-13]. CPAs have shown to be greater in lateral muscles, especially in older faller and nonfaller patients [10].

The postural control mechanism is a spatially and temporally dynamic process dependent upon the external environment and system status [14-16]. Control characteristics may change over short periods of time, perhaps adapting to updates from sensory information [12]. Impaired sensory and motor systems increase the central processing load to maintain postural balance. The attentional resources may not be available due to cognitive impairments and central processing disorders. Patients with AD demonstrate a decline in postural control due to cortical deficits associated with impairments to sensory organization, such as suppression of visual or auditory distractions, dual-tasking, and diversion of attention to another focus [17-19].

Numerous studies have also reported that postural responses occur on two distinct timescales: a fast (high-frequency) open-loop control and a slower (low-frequency) corrective feedback-based control $[19,20]$. Despite the lack of a clear distinction between slow and fast sway components, the lower frequencies can be attributed to the inertial properties of the oscillating mass and the high-frequency components are the net contribution of irregular voluntary and involuntary muscle activity, as well as the product of multisensory feedback integration [20]. In a previous study [21], we shed light on the dynamic control of posture, in particular CPAs, by using a time-frequency analysis of CPAs in a changing virtual reality (VR) setting in idiopathic Parkinson's disease (IPD) patients. CPAs were actuated in two different frequency bands: a low-frequency band (LB; $0.3-1.5 \mathrm{~Hz}$ ), representing the mechanical properties of oscillation on postural correction, and a high-frequency band (HB; $1.5-3.5 \mathrm{~Hz}$ ), reflective of the higher cognitive strategies of postural correction.

An appropriate nonstationary technique, such as time-frequency analysis, should be employed to characterize the existing dynamic variations [22]. The concept of time-frequency distributions (TFDs), as functions of both time and frequency, was introduced to circumvent the limitations intrinsic to stationary signal analyses [23]. One of the first solutions presented implements an extension to traditional Fourier analysis: the short-time Fourier transform. This estimates the energy spectrum (spectrogram) using a sliding window, thus assuming local stationarity of the signal. The resulting spectrogram depends on the window size, which is responsible for the trade-off between time and frequency resolutions. The bigger the window size, the better the frequency resolution and the ability to detect low-frequency components that are characteristic of postural adjustments. On the other hand, it sacrifices a 
Gago et al:: Compensatory Postural Adjustments in an Oculus Virtual Reality

Environment and the Risk of Falling in Alzheimer's Disease

large amount of time information, which is especially critical when analyzing short-duration signals. This is the major limitation to spectrograms, making them unsuitable for center of mass (COM) data analysis $[22,24]$. The minimum mean cross-entropy (MMCE) method combines information from a finite set of spectrograms computed with different window sizes, producing a much better approximation of the time-varying spectrum than any of these individual spectrograms. Investigations with the MMCE method have pointed out its low computational demand and ability to closely approximate positive TFDs of the Cohen-Posch class, which are the most appropriate for real-world signal analysis [25].

In the last years, the impact of visual perturbation on postural adjustments has been widely explored. In AD patients with a previous history of falls, postural control seems to be more vulnerable to the loss of visual input, increasing several postural sway parameters [26]. Susceptibility to visual stimulation has been studied in several conditions such as with a visual focus on differently distanced targets [27] or exposal to a moving surround, which in most of the recent studies was implemented through an immersive virtual scene allowing for a better perception of the induced motion. This VR creates an illusion that puts the subject in a place other than where he/she physically is [28]. Besides inducing the illusion of self-motion, the moving visual surround conflicts with perceptions from the somatosensory and vestibular systems, since the body does not actually move. As a consequence, the body generates CPAs in the direction of the visual perturbation $[29,30]$, which affects elderly and impaired subjects on a larger scale $[12,31]$.

Some studies pointed out the sensitivity of the vertical component to detect changes in postural balance [21, 32]. Settings comprising force platforms restrict their analyses to the 2D plane beneath the feet of the evaluated subject. If a vertical visual motion is evaluated, these data might not be able to properly reflect postural responses [29]. Inertial measurement units have emerged as tools complementary to conventional posturography, providing additional information about body tilt and orientation with the advantages of lower cost, reduced size, and portability [33, 34]. Given the prevalence of falls and the risks associated with them among patients with $\mathrm{AD}$, the present study was designed in a way to submit participants to visual downward displacements mimicking the illusion of falling. Patients with AD perform poorly in tests of shifting visual attention or incongruent visual stimuli, suggesting a decreased ability to suppress the conflict induced by visual stimulation [17]. This finding motivated the design of an experiment where participants are immersed in a VR with unexpected visual displacements.

The primary objective of this research was to study postural adjustment mechanisms in $A D$, evaluating visually induced CPAs in a changing VR scenario in healthy subjects and AD patients by means of kinematic and time-frequency analyses on inertial measurement unit records. As a second objective, we aimed to relate the CPA profile to the risk of falling in AD by considering distinct groups of patients with $\mathrm{AD}$ (i.e. patients with and without a previous history of falls).

\section{Methodology}

\section{Subjects and Clinical Assessment}

The study protocol was approved by the local ethics committee, and the study was carried out in accordance with the Declaration of Helsinki. Written informed consent was obtained from all participants in the study. Twenty-one AD patients were consecutively recruited from our dementia outpatient consult who fulfilled the criteria for probable AD according to the Diagnostic and Statistical Manual of Mental Disorders-IV (DSM-IV) and the National Institute of Neurological and Communicative Disorders and Stroke/Alzheimer's Disease and Related 

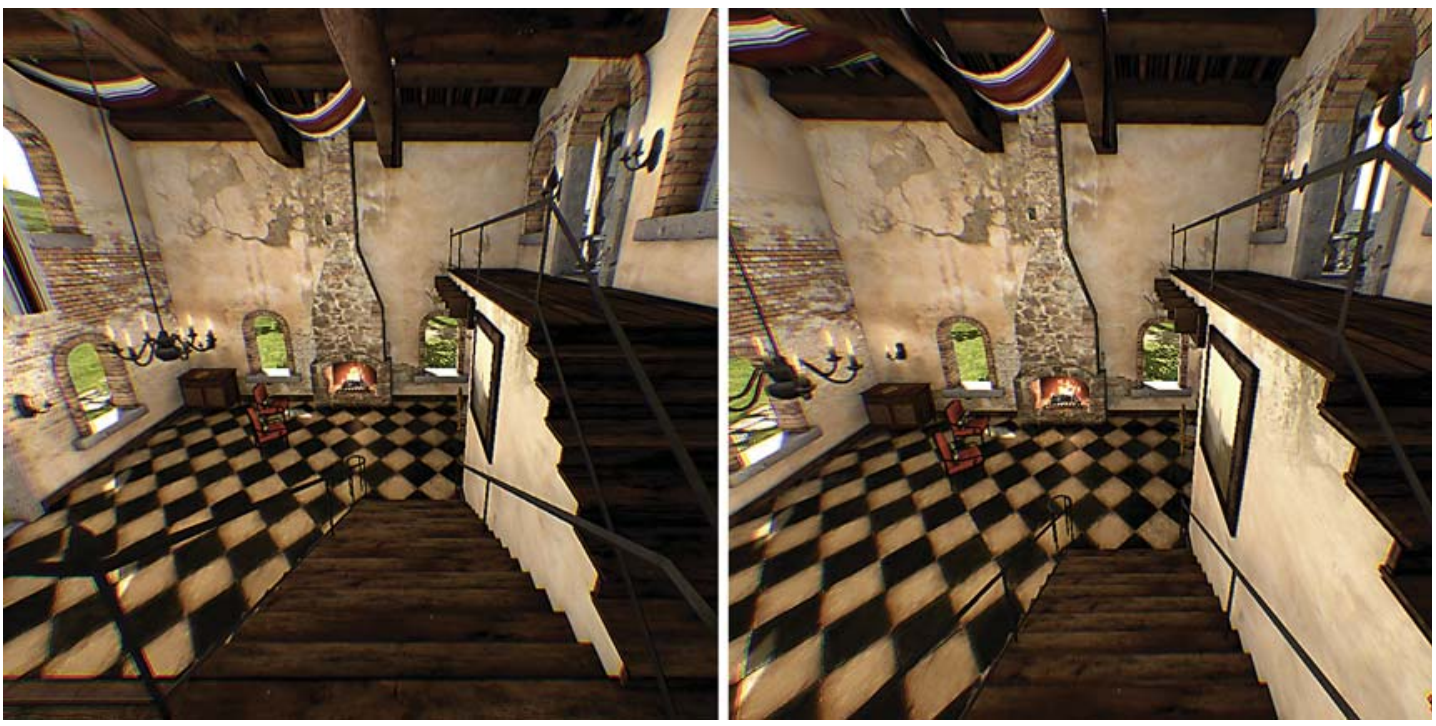

Fig. 1. Virtual environment created via Oculus Rift goggles, with the two translation scenarios: at the top of the staircase (left) and, after a visual displacement of $1.17 \mathrm{~m}$ along the vertical axis, translation to the middle of the stairs (right). Reproduced from Yelshyna et al. [21] (by permission of Darya Yelshyna).

Disorders Association (NINCDS/ADRDA) [35] (a score of 1 on the Clinical Dementia Rating scale). The control group consisted of the same 19 healthy subjects (controls) already used for a previous publication [21]. The exclusion criteria were orthopedic, musculoskeletal, and vestibular disorder, significant auditory deficit, and alcohol abuse and somatosensory deficit.

The recruited patients had normal vision dispensing with the use of glasses or contact lenses to correct their vision. Assessment of falls in the 12 months prior to assessment was carried out via fall calendars. A fall was defined as an unexpected event in which a participant comes to rest on the ground, the floor, or a lower level [36]. AD patients were recorded as fallers (AD fallers) if they had had at least one fall in the previous 12 months, in contrast to nonfallers (AD nonfallers). The collected variables consisted of demographic (gender, age, and education) and anthropometric data (weight, height, and body mass index). Clinical data were also collected - including years of disease duration and a neuropsychological examination using the Portuguese version of the Montreal Cognitive Assessment (MoCA) with scores normalized to the Portuguese population [37] - no more than 1 month prior to the kinematic assessment. Levels of education were categorized by years of schooling as follows: 0 (analphabetic), 1 (1-4 years), 2 (5-9 years), 3 (10-12 years), and 4 (>12 years).

\section{Apparatus and Postural Tasks}

For this study, we implemented the same methodology as previously reported in the study on Parkinson's disease [21]. One sensing module, harboring an 8051 microprocessor embedded in a CC2530 Texas Instrument SoC (system on chip), and a wearable inertial measurement unit (MPU6000; triaxial accelerometer and gyroscope), operating with a sample rate frequency of $118 \mathrm{~Hz}$ on an SD card [38], were attached to the COM, located at 55\% of the patient's height above the ground.

The subjects were submitted to a realistic visual scene with 3D depth information by wearing Oculus Rift goggles - a VR headset device with a 100-degree field of view. Visual focus and eye width settings were adjusted for each participant to display a clear stereoscopic 3D image. Tuscany Demo (fig. 1) was the chosen scenario for subject evaluation. Several objects 
Gago et al:: Compensatory Postural Adjustments in an Oculus Virtual Reality

Environment and the Risk of Falling in Alzheimer's Disease

were embedded in the virtual scene: floor, roof, stairs, fireplace, chandelier, window, and door. In this virtual environment, the subjects stood at the top of the staircase, with the purpose of performing unexpected, visually induced motions and evaluation of the response to the illusory perception of falling (translation down the stairs). While performing the tasks, the subjects were instructed to keep standing still, barefoot, with the medial aspects of their feet touching each other, with their arms hanging at their sides and using a safety trunk belt. The subjects were instructed not to abandon this position and, if need be, to make a corrective adjustment by bending their knees.

Each participant underwent a preparatory phase. They were asked to identify and search for several objects embedded in the VR setting so that they could feel fully immersed in the environment. Inside the virtual environment, the subjects stood at the top of the staircase and were issued to focus their attention on the first stair below their feet. Approximately $10 \mathrm{~s}$ later, the scenario instantaneously moved down, creating a visual displacement, translating the subject to the middle of the stairs. After another $10 \mathrm{~s}$, the scenario moved upwards, placing the subject back on the top of the stairs, until the next trial. After experiencing several translations, the subjects were asked to state if they felt that they were virtually pushed onto the middle of the stairs, as they would have experienced in real life. This procedure was repeated several times, with a minimum of a 20 -second gap between trials and a minimum of 5 preparatory trials per subject, so as to guarantee immersion in the VR setting, as assessed by the subjects' answers about having the feeling of subjective immersion in VR. Previous studies, also focusing on perturbations, have shown that our intertrial time intervals were adequate, as the participants were able to change sets from one condition to another [39].

With the same methodology as for the preparatory phase, a total of 5 effective downward trials per subject (10 s duration) were selected as the object of subsequent analysis. The neurologist was responsible for triggering the downward translation in a manual and random fashion, thus averting adaptation and a learning effect and keeping individuals unaware of the exact moment of the upcoming event. The visual perturbation in VR elicited no need for a corrective step in either group. The translation in the virtual environment corresponded to approximately $1.17 \mathrm{~m}$ of displacement along the vertical axis.

\section{Data Analysis}

No clear procedures have yet been established for accelerometer data processing, but the main energy content of human movement is held below $3.5 \mathrm{~Hz}[33,40]$. For this reason, the raw acceleration signals were filtered with a zero-phase low-pass Butterworth filter with a $3.5-\mathrm{Hz}$ cutoff frequency. Due to the abdominal placement of the sensor, acceleration signals went through additional high-pass filtering in order to eliminate interference that might be caused by the act of breathing. Considering a normal respiratory frequency in adults (i.e. 18 breaths/min [41]), we applied a zero-phase high-pass Butterworth filter with a $0.3 \mathrm{~Hz}$ cutoff frequency. A more precise evaluation of visual destabilization is achieved when the subject is focused and unaware of the upcoming event. Tasks that require precise eye fixation, as in a visual search for objects in the surrounding environment, appear to decrease sway variability [42]. In order to identify this increased attentional demand during quiet stance, we computed the TFD's total power in the HB $(1.5-3.5 \mathrm{~Hz}) 4$ s prior to the visual stimulus for all trials and selected the one with the lowest value. This trial was considered most representative of the response to visual stimulation and was evaluated by means of kinematic and time-frequency analyses.

Kinematic Analysis

Descriptive statistics such as standard deviation, maximum, minimum, and range were computed for the acceleration signals for the three axes, as well as for the orientation and 
Gago et al.: Compensatory Postural Adjustments in an Oculus Virtual Reality

Environment and the Risk of Falling in Alzheimer's Disease

displacement estimates. Also, the common features related to COM excursion (e.g. sway area and sway path) were computed from the estimated COM position [11]. Kinematic measures were computed from 8-second time segments immediately after the onset of the selected visual downward-moving stimulus.

Another version of the accelerometer signal - one that takes lower frequency components into account - was considered in order to characterize body orientation and to approximate its displacement [26, 34]. For this purpose, both the raw acceleration and gyroscope signals underwent zero-phase low-pass Butterworth filtering with 0.5- and 2-Hz cutoff frequencies, respectively. A detailed explanation of the methodology used to obtain body orientation - pitch and roll angles - and an estimate of COM displacement and acceleration can be found in our previous study [26].

Time-Frequency Analysis

In a previous study [21], we shed light on the dynamic control of posture, in particular CPAs, by using a time-frequency analysis of CPAs in a changing VR setting with IPD patients. CPAs were actuated in two different frequency bands: LB $(0.3-1.5 \mathrm{~Hz})$, representing the mechanical properties of oscillation on postural correction, and $\mathrm{HB}(1.5-3.5 \mathrm{~Hz})$, reflective of higher cognitive strategies of postural correction.

For each subject, we extracted time segments including all trials with a margin of $10 \mathrm{~s}$ prior to the first and after the last stimulus. The time-varying spectrum was estimated by an MMCE combination Hann window of spectrograms computed with windows of 15, 31, and 127 samples [25]. Using this large time segment including all the trials performed, it is possible to obtain a TFD with a high time and frequency resolution. Afterwards, the relevant trial was identified, and only the corresponding portion of the TFD $4 \mathrm{~s}$ before and $8 \mathrm{~s}$ after stimulus onset was extracted to perform further computations (fig. 2). The Time-Frequency Toolbox (v1.2) for MATLAB ${ }^{\odot}$ provided the function for MMCE computation.

A quantitative description of dynamic TFD behavior can be achieved by defining several time-dependent parameters, including instantaneous average power $[43,44]$. The instantaneous average power allows monitoring sudden changes due to postural adjustments. This feature was computed for each of the frequency bands considered (HB and LB) for 3 time intervals: the 4-second interval prior to stimulus onset ( -4 to $0 \mathrm{~s}$ ) and two 4 -second intervals after stimulus onset (0-4 s and 4-8 s). All data processing and feature computation were performed with a custom-made MATLAB ${ }^{\odot}$ code.

\section{Statistical Analysis}

Gender comparisons were analyzed by the $\chi^{2}$ Fisher exact test. Given the small number of subjects, the intergroup statistical analysis was carried out with a nonparametric KruskalWallis test, with a pairwise post hoc analysis with Dunn's test. The computations were performed with a Monte Carlo simulation with a 99\% confidence level. The Wilcoxon matchedpair test was used for assessing the intragroup magnitude of changes for the separate band powers of TFD analysis. Statistical analyses were conducted with the software SPSS v.20.0.

\section{Results}

\section{Subjects}

Twenty patients with AD (11 fallers, 9 nonfallers) and 19 controls were included in this study. The demographic and anthropometric characteristics of the three groups are summarized in table 1 . The groups were equally matched according to demographic and anthropometric characteristics. AD patients, as expected, presented with lower scores on the MoCA 

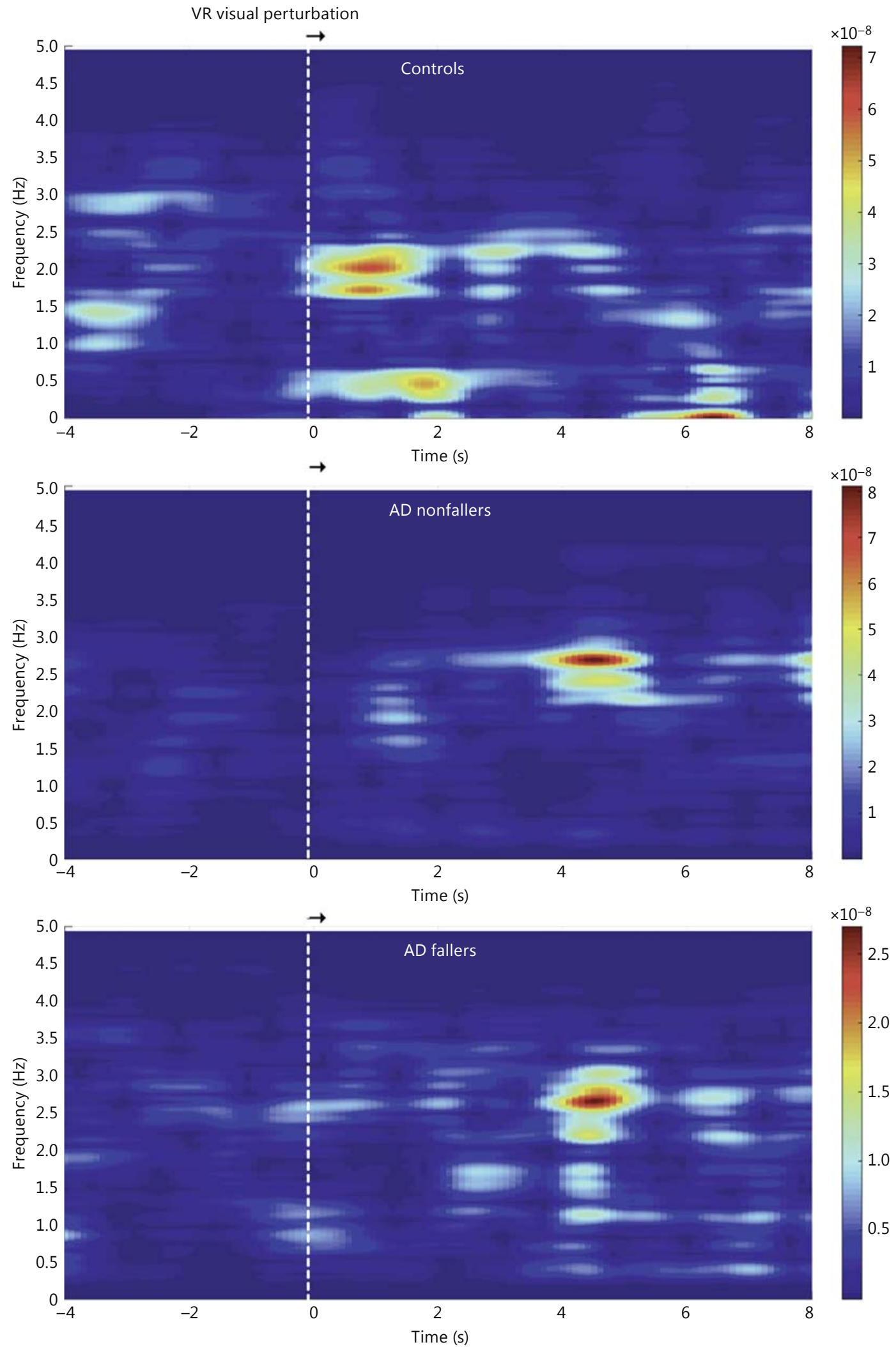

Fig. 2. Examples of TFDs obtained from the representative trial for each of the groups studied: controls, $A D$ fallers, and AD nonfallers. The visual downward perturbation occurred at $0 \mathrm{~s}$. 
Gago et al.: Compensatory Postural Adjustments in an Oculus Virtual Reality Environment and the Risk of Falling in Alzheimer's Disease

Table 1. Demographic and clinical data on controls, AD fallers, and AD nonfallers

\begin{tabular}{lccc}
\hline & $\begin{array}{l}\text { Controls } \\
(\mathrm{n}=19)\end{array}$ & $\begin{array}{l}\text { AD fallers } \\
(\mathrm{n}=11)\end{array}$ & $\begin{array}{l}\text { AD nonfallers } \\
(\mathrm{n}=9)\end{array}$ \\
\hline Gender (female/male), $\mathrm{n}$ & $11 / 8$ & $9 / 2$ & $5 / 4$ \\
Age, years & $71(51-78)$ & $76(66-82)$ & $75(61-82)$ \\
Weight, kg & $75(56-107)$ & $66(42-80)$ & $66(54-86)$ \\
Height, m & $1.63(1.49-1.79)$ & $1.51(1.41-1.77)$ & $1.58(1.44-1.69)$ \\
Body mass index & $28(22.9-33.4)$ & $26(18.8-34.8)$ & $27(21.9-39.7)$ \\
Level of education & $1(0,2)$ & $1(0,2)$ & $1(0,1)$ \\
MoCA score* & $24(18-30)$ & $9(5-19)$ & $12(7-17)$ \\
Disease duration, years & - & $3(2-4)$ & $3(3-5)$
\end{tabular}

Data are presented as medians (min.-max.). For levels of education, see Methodology. * Statistically significant difference on Kruskal-Wallis intergroup comparison.

( $p<0.01$; controls vs. AD fallers: $p<0.001$; controls vs. AD nonfallers: $p<0.001$ ). However, there was no statistical difference in MoCA score or disease duration between the two AD groups.

\section{Kinematic Features}

The vertical acceleration signal demonstrated a greater power in discriminating AD fallers from controls. The AD faller group presented a higher range of acceleration on the $\mathrm{z}$-axis (controls vs. AD fallers: $\mathrm{U}=38.0, \mathrm{z}=-2.862, \mathrm{p}=0.003$ ), with a higher mean acceleration $(\mathrm{U}=53.0, \mathrm{z}=-2.216, \mathrm{p}=0.026)$, root mean square acceleration $(\mathrm{U}=57.0, \mathrm{z}=-2.044, \mathrm{p}=$ $0.042)$, and average acceleration magnitude ( $U=53.0, z=-2.216, p=0.026)$ (fig. 3 ).

\section{Time-Frequency Distributions}

The TFD analysis reflected pronounced differences in the frequency patterns of CPAs in response to visual perturbation, perceived at the separate power band levels in the various time intervals before and after visual perturbation. The control, AD faller, and AD nonfaller groups perceived and reacted to visual perturbations with different patterns (table 2).

In comparison to the controls, the AD nonfaller group had significantly higher values of power of CPAs only in the $\mathrm{HB}$, and only just before visual perturbation $(-40 \mathrm{~s})$. In contrast, the AD faller group had significantly higher values of power of CPAs both in the LB $(<1.5 \mathrm{~Hz})$ and the $\mathrm{HB}(>1.5 \mathrm{~Hz})$ during all time intervals (fig. 4, 5), i.e. before $(-40 \mathrm{~s})$ and immediately after visual perturbation ( $0-4 \mathrm{~s})$ as well as at the end (4-8 s).

In the intragroup analysis of the pattern of progression of CPAs in the different time intervals, none of the groups had significant changes in power in the LB. In the HB, only the control group had a significant increase in power immediately after visual perturbation $(-4$ to 0 vs. $0-4 \mathrm{~s})$, a reaction also present towards the end (0-4 vs. 4-8 s). In contrast, the AD faller and nonfaller groups had a delayed reaction, with a significant increase only in the last interval (0-4 vs. $4-8 \mathrm{~s})$.

\section{Discussion}

Different central postural control mechanisms may be continuously and dynamically taking place, and therefore TFD analysis may provide further information on the postural reaction and susceptibility to visual perturbation. Some studies have demonstrated that 


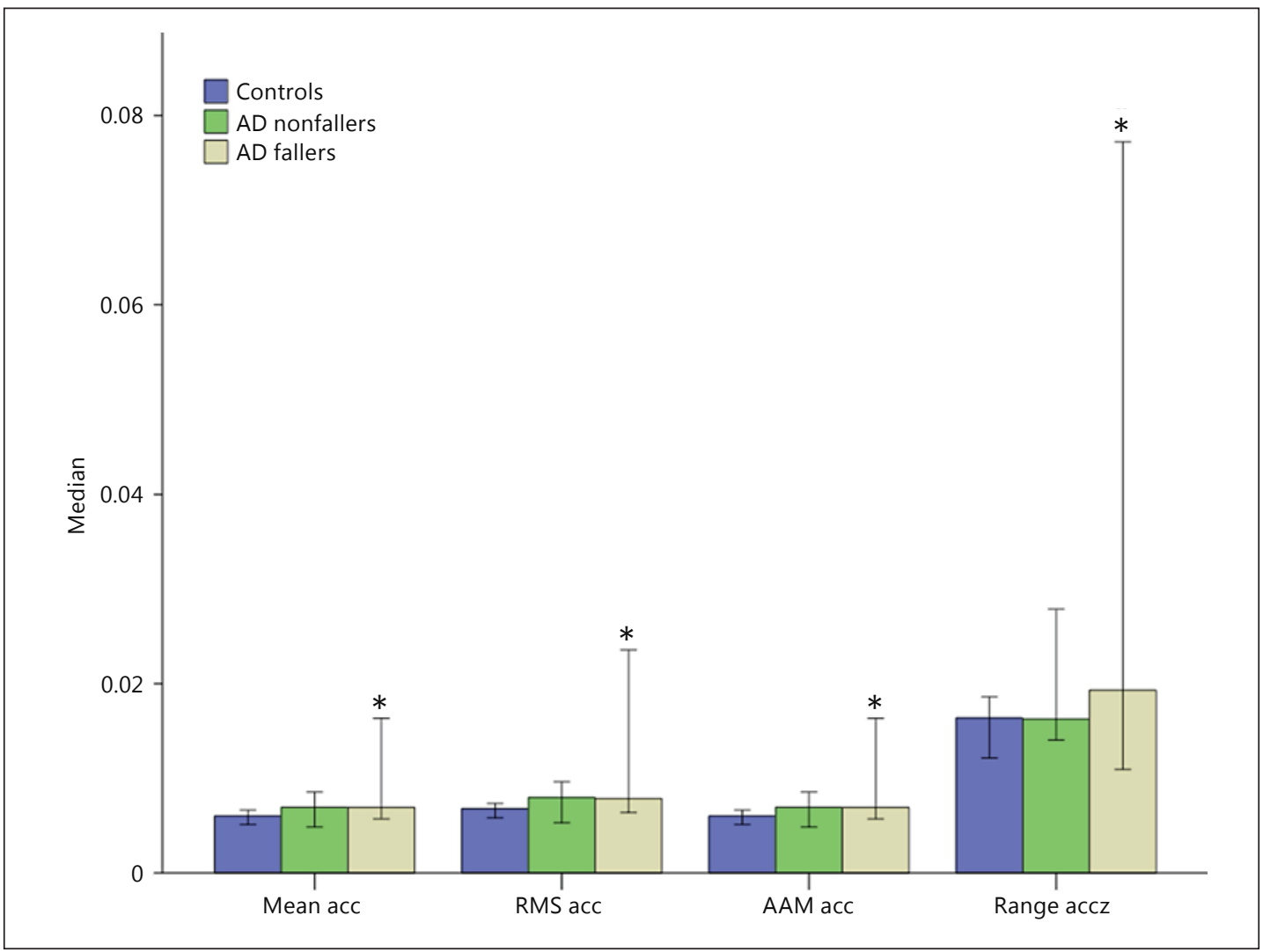

Fig. 3. Errors bars ( $95 \%$ confidence intervals) of the median values of the acceleration measures mean acceleration (acc), root mean square (RMS) acceleration, average acceleration magnitude (AAM), and range of acceleration on the z-axis (Range accz) for healthy subjects (controls), AD fallers, and AD nonfallers. The AD faller group had statistically significantly $(* p<0.05)$ higher values versus the control group.

postural control takes place on two distinct time scales: a fast (high-frequency) open-loop control and a slower (low-frequency) corrective feedback-based control [21, 45]. Indeed, LB components seem to be largely dictated by the inertial properties of the oscillating mass of the subject, reflecting a more mechanical automatic oscillation mechanism of stabilization and posture control that is dependent on the stiffness of the musculotendinous structure. In contrast, HB oscillatory components of sway are more likely to represent the lump sum of irregular voluntary and involuntary muscle activity and multisensory feedback integration [46]. Moreover, performance on cognitive tasks has been shown to be only influenced by variability in the fast sway components [20]. In our study, the dissonance between visual input and somatosensory perceptions (proprioceptive and vestibular) provoked by VR induced CPAs. These CPAs were most evident immediately after the visual environmental scenario change but carried on for $8 \mathrm{~s}$ (fig. 4, 5). In this study, using the same methodology as in our previous study [21], we showed that AD fallers clearly presented CPAs with higher values of acceleration and with a different pattern of distribution of power in the LB and HB.

CPAs in the $L B$

Our VR paradigm, with a translation within the virtual environment of $1.17 \mathrm{~m}$ along the vertical axis, did not elicit any significant destabilization that required CPAs in the LB in any of the groups. Nevertheless, AD fallers had more pronounced CPAs in the LB irrespective of 


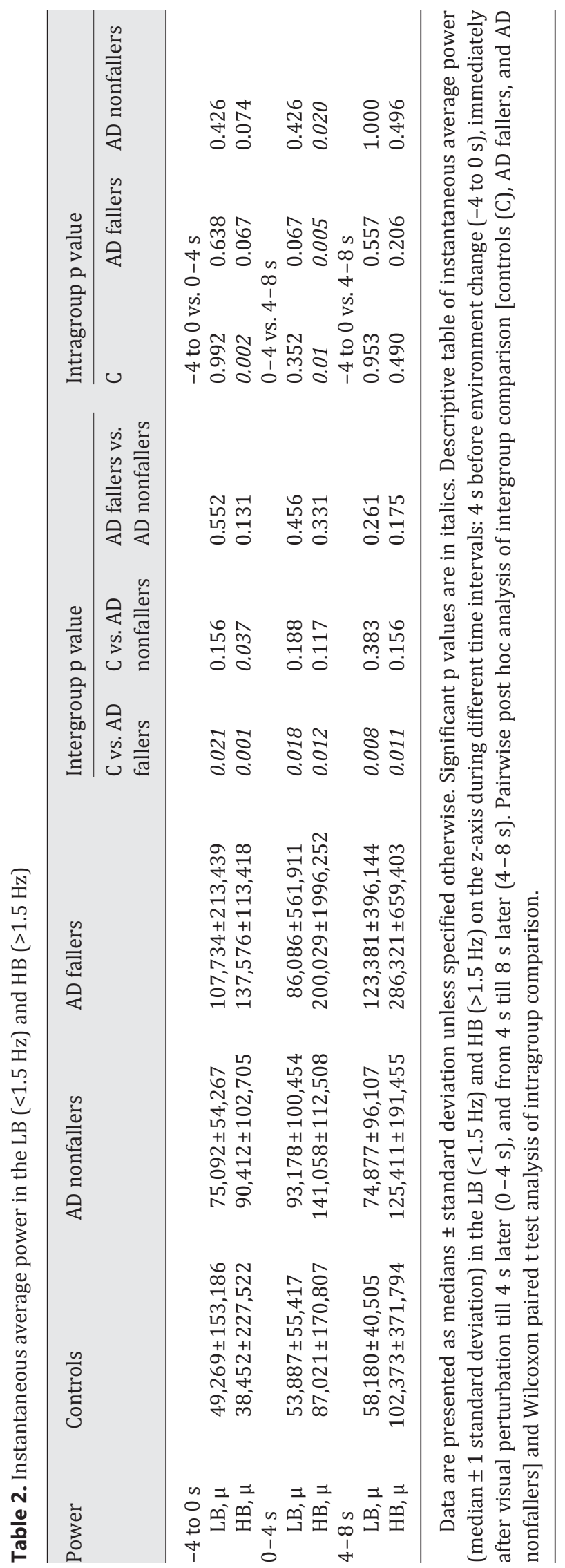




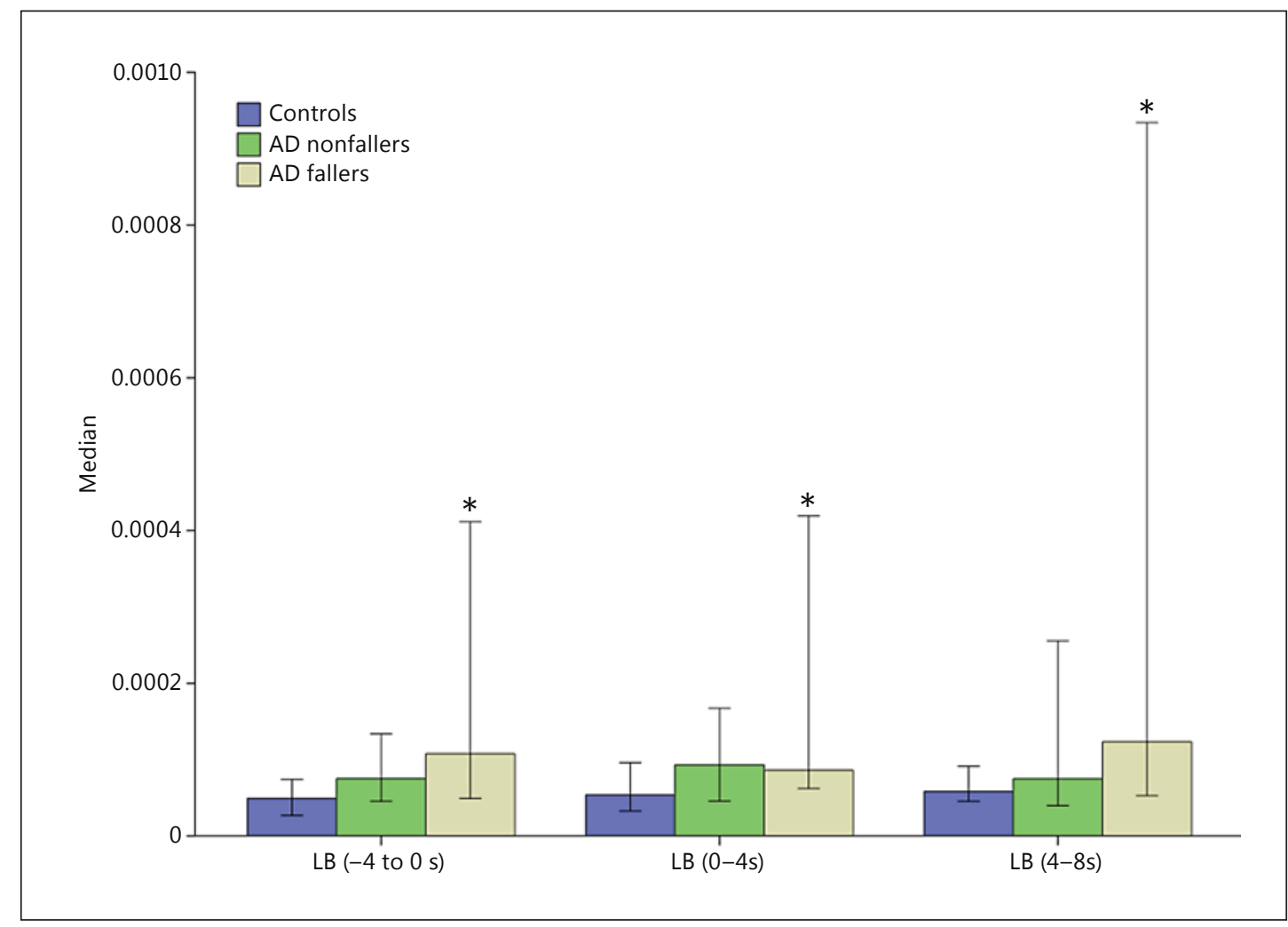

Fig. 4. Errors bars ( $95 \%$ confidence intervals) of the median values of the power in the LB $(<1.5 \mathrm{~Hz})$ for healthy subjects (controls) and AD patients (fallers and nonfallers), averaged over 4-second intervals ( -4 to $0,0-4$, and $4-8 \mathrm{~s})$. There was a statistically significant difference $(* p<0.05)$ on Mann-Whitney intergroup comparison between controls and AD fallers. There were no statistically significant changes between the different intervals in any group.

the different time interval and response to visual perturbation. As already stated, the LB reflects a subconscious automatic oscillation mechanism of stabilization and posture control that is dependent on the stiffness of the musculotendinous structure. Thus, our findings may reflect an inherently higher instability of $\mathrm{AD}$ faller patients, requiring greater actuation of continuous restoring and compensatory forces, provided by the slow acceleration components.

\section{CPAs in the $H B$}

With due limitations to any extrapolation to real-life scenarios, our moving, immersive VR environment was a useful tool for analyzing CPAs in response to visual perturbations and to further comprehend the underlying mechanisms of corrective postural adjustment. In our VR environment, several cognitive, emotional, and sensorial conflicts occurred simultaneously. After visual perturbation, all subjects required corrective adjustments in the HB, thus corroborating the role of higher cognitive processes in postural control.

We found that healthy subjects had an immediate response to the visual perturbation and an inherent increase in CPAs in the HB. In contrast, the AD faller and nonfaller groups had a delayed reaction, with a significant increase only during the last interval, after $4 \mathrm{~s}$. Also, besides responding with a time lag in reaction to the visual perturbation, $\mathrm{AD}$ fallers had higher CPAs in the HB. This clearly reflects their higher vulnerability to visual perturbation and the 


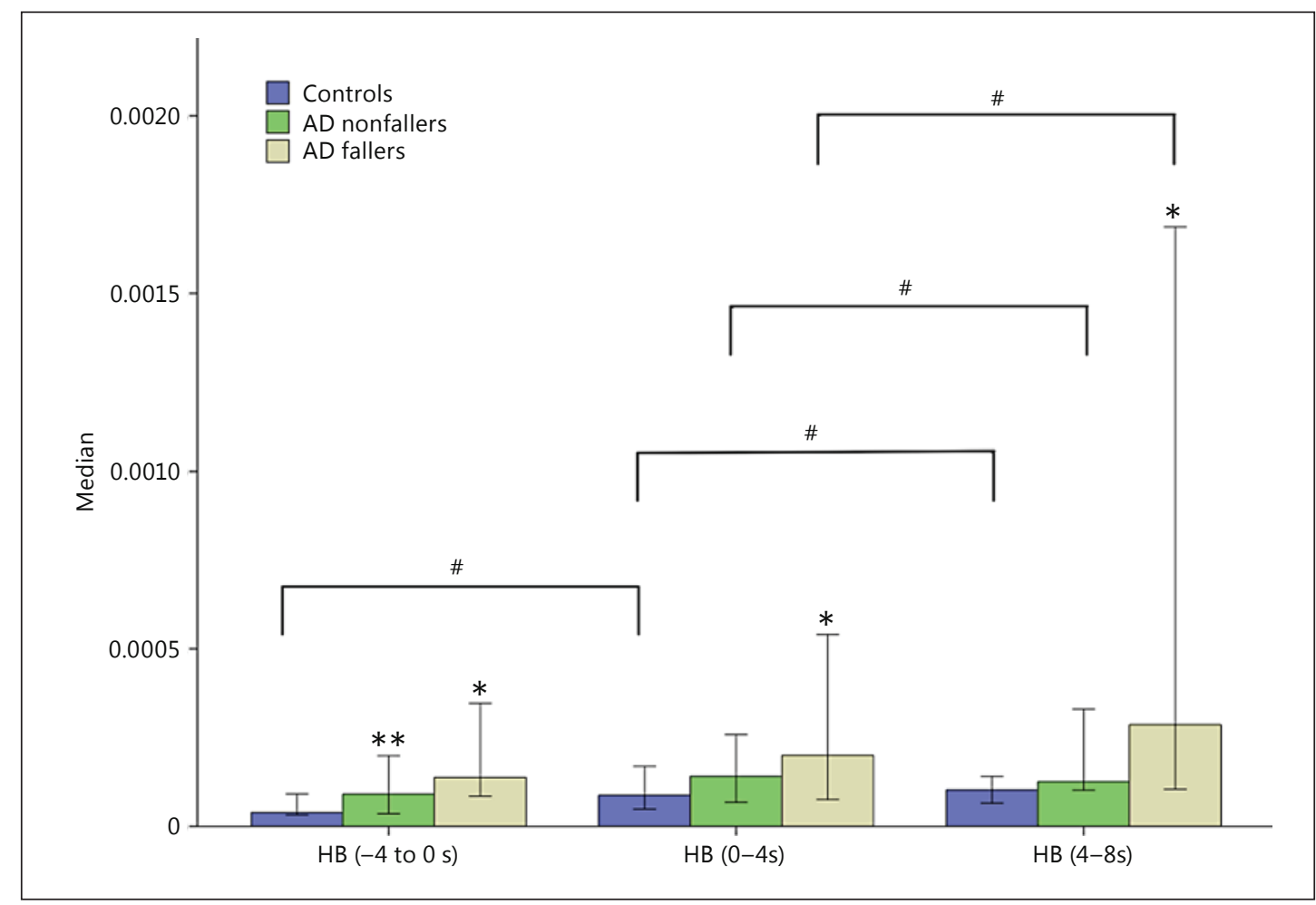

Fig. 5. Errors bars (95\% confidence intervals) of the median values of the power in the HB $(>1.5 \mathrm{~Hz})$ for healthy subjects (controls) and AD patients (fallers and nonfallers) averaged over 4-second intervals ( -4 to 0, 0-4, and 4-8 s). There was a statistically significant difference on Mann-Whitney intergroup comparison between controls and AD fallers ( $\left.{ }^{*} \mathrm{p}<0.05\right)$, and between controls and AD nonfallers $(* * \mathrm{p}<0.05)$. Brackets with a hash mark $\left({ }^{\#} \mathrm{p}<0.05\right)$ represent significance on intragroup Wilcoxon paired t tests. The control group had a statistically significant increase during the first interval ( -4 to $0 \mathrm{~s}$ ). All groups had a statistically significant increase between the second (0-4 s) and the last interval (4-8 s).

need to produce corrective adjustments to external perturbations in order to maintain the COM within the limits of stability [17-19].

Several hypotheses can be raised to explain this profile of delayed and higher CPAs to external perturbations in AD fallers. Postural control that takes place in the HB probably reflects a more central cognitive and volitional mechanism of postural control [20,45]. With aging, the attentional demands of postural control increase as sensory information decreases, and the inability to allocate sufficient attention to postural control under multitasking conditions may be a factor contributing to imbalance and falls in some older adults [3]. Patients with AD demonstrate a decline in postural control due to cortical deficits associated with impairments to sensory organization, such as suppression of visual or auditory distraction, dual-tasking, and diversion of attention to another focus [17-19]. Response inhibition, an executive cognitive function, allows one to ignore irrelevant sensory inputs, overcome primary reflexes, filter out distractions, respond discriminatively to important features in the environment, and focus on postural stability [47]. The impaired ability of the CNS to quickly reweight sensory dependence in $\mathrm{AD}$ (even when the peripheral sensory system is intact) in response to dynamic perturbations in the sensorial environment during daily life increases the risk of falling [17]. Individuals who have limited cognitive processing due to neurological impairments, such as AD patients, may need to use more of their available cognitive processing 
Gago et al:: Compensatory Postural Adjustments in an Oculus Virtual Reality

Environment and the Risk of Falling in Alzheimer's Disease

to control posture [48], increasing their susceptibility to falls. In fact, the slower reaction time to postural perturbations in AD has been associated with a higher risk of falls $[49,50]$, corroborating our results.

AD nonfallers and fallers - even if they may appear similar on initial clinical and demographic evaluation - may have different cognitive resources available for postural control and, ultimately, a higher risk of falling. In comparison to healthy subjects, our visual scenario of imminent and unpredictable falls could have elicited higher COM imbalances in AD patients, demanding higher CPAs in the HB. A recent study using VR and gait analysis in IPD patients provided evidence that cognitive dysfunction, such as anxiety, interfered with proper information processing [51]. The reaction to an unexpected visual perturbation, such as in our VR paradigm, required that body schema self-perception and motor programming be preserved and adequately updated in the temporoparietal cortex. The finding of delayed and more exacerbated CPAs in the HB in the AD faller group may be a consequence of a more severe atrophy of the temporoparietal cortex. In the absence of brain imaging in our study, this hypothesis deserves further exploration. Interestingly, some studies have reported that the hippocampus uses vestibular information for spatial memory and navigation, and that balance impairment could be related to reduced hippocampal performance [52]. Another point of view is that the delayed reaction of our AD patients when facing the visual-vestibulosomatosensory conflict posed by the VR paradigm could also be due to impairments of visual motion, shape [53], and depth perception [54] reported in AD. Furthermore, threat-related factors influence the neuromechanical postural responses to an unpredictable perturbation, and these responses may be facilitated in younger healthy adults [55]. Thus, the emotional state, such fear of and anxiety about falling, may explain different CPA responses among AD patients, in particular in AD fallers.

\section{Study Limitations}

Our small sample impairs robust statistical inference, and thus our results have to be taken with caution. Our exclusion criteria - in particular the absence of visual, musculoskeletal, vestibular, and auditory deficits - clearly contributed to the smallness of our sample. These deficits are extremely prevalent in geriatric populations and, if not excluded, would have been an even greater confounder for the interpretation of CPAs. Studies that showed distinct timescale mechanisms for postural control, a low and a fast one, defined cutoffs of 0.3 $\mathrm{Hz}$ for center-of-pressure position data [20,45]. Postural analysis with accelerometer data requires an assessment in the range of frequencies from 0.3 to $3.5 \mathrm{~Hz}$ to determine which frequency would yield a proper discrimination between slow and fast components of CPAs. In this study, to distinguish between low- and high-frequency domains of CPAs, a band separation at $1.5 \mathrm{~Hz}$ was chosen, since upon visual inspection it appeared to yield representative and discriminative results, using the same methodology as in our previous work [21]. Nevertheless, this decision was purely subjective and should be explored further. The clear instruction to the subjects to maintain a fixed posture, with their feet together, and to use knee-bending as a corrective strategy may have potentially played a greater role in the prominent differences in CPAs along the vertical axis (z-axis) observed [21]. This is important, because in a real-life scenario, forward or lateral step correction, or the use of the lateral muscles, usually happens in free open space. Moreover, CPAs have been shown to be greater in the lateral muscles, especially in older faller and nonfaller patients [10]. As already mentioned, executive functions have an important role in CPAs, and decreased executive functions are associated with worse performance on functional measures of balance [56]. This could be further explored in a broader neuropsychological examination in a future study. 
Gago et al.: Compensatory Postural Adjustments in an Oculus Virtual Reality

Environment and the Risk of Falling in Alzheimer's Disease

\section{Conclusion}

The impaired ability of the CNS to quickly reweight sensory dependence in AD (even when the peripheral sensory system is intact) in response to dynamic perturbations in the sensorial environment during daily life increases the risk of falling. Our VR paradigm, which produced a sensorial dissonance between a changing visual environment and static sensorial inputs, induced significant CPAs in healthy subjects and AD patients, especially in the HB. Different postural adjustment mechanisms were at work in the LB and HB. The postural adjustment mechanisms in the LB reflect the inertial mechanical properties of the oscillating mass of the body, whereas in the HB, they represent higher cognitive multisensory feedback integration and reaction to the ongoing scenario of changing visual inputs. Our AD fallers presented a higher power of CPAs in the LB, reflecting an inherently higher instability of AD fallers and the requirement of continuous restoring and compensatory forces, provided by the slow acceleration components. The AD patients presented delayed CPAs in the HB, with a significant time lag to visual perturbation in comparison to the healthy subjects, with the AD fallers needing greater CPAs. This delayed profile of CPAs to visual perturbations and misjudgments of sensory feedback may be a reflection of slower reaction times due to different cognitive resources and/or errors, including body schema self-perception in the temporoparietal cortex, visual motion and depth perception impairments, or a direct consequence of different emotional states such as fear and anxiety and a higher risk of falling.

Analyses of postural adjustment kinematics in different VR settings and paradigms, translating the more complex real-life challenges into a standardized and controlled mode, may allow better comprehension of the different (mechanical, sensorial, and higher cognitive) systems that play a role in postural control. As falls are very prevalent and an important issue in $\mathrm{AD}$, further studies are needed, with larger samples, to prove that kinematic analysis of CPAs is a useful tool for clinical practice in identifying patients with higher risks of falling and, therefore, allowing the implementation of preventive measures.

\section{Disclosure Statement}

The Centro ALGORITMI was funded by the FP7 Marie Curie ITN Neural Engineering Transformative Technologies (NETT) project. The authors have no conflicts of interest to report.

\section{References}

1 Kannus P, Sievänen H, Palvanen M, Järvinen T, Parkkari J: Prevention of falls and consequent injuries in elderly people. Lancet 2005;366:1885-1893.

-2 Etman A, Wijlhuizen GJ, van Heuvelen MJ, Chorus A, Hopman-Rock M: Falls incidence underestimates the risk of fall-related injuries in older age groups: a comparison with the FARE (Falls Risk by Exposure). Age Ageing 2012;41:190-195.

-3 Shumway-Cook A, Woollacott M: Attentional demands and postural control: the effect of sensory context. J Gerontol A Biol Sci Med Sci 2000;55:M10-M16.

4 Grossman H, Bergmann C, Parker S: Dementia: a brief review. Mt Sinai J Med 2006;73:985-992.

5 Scarmeas N, Hadjigeorgiou GM, Papadimitriou A, Dubois B, Sarazin M, Brandt J, Albert M, Marder K, Bell K, Honig LS, Wegesin D, Stern Y: Motor signs during the course of Alzheimer disease. Neurology 2004;63:975982.

6 Buchner DM, Larson EB: Falls and fractures in patients with Alzheimer-type dementia. JAMA 1987;257:14921495.

7 Kanekar N, Aruin AS: Aging and balance control in response to external perturbations: role of anticipatory and compensatory postural mechanisms. Age (Dordr) 2014;36:9621. 
Gago et al:: Compensatory Postural Adjustments in an Oculus Virtual Reality

-8 Park S, Horak FB, Kuo AD: Postural feedback responses scale with biomechanical constraints in human standing. Exp Brain Res 2004;154:417-427.

9 Alexandrov AV, Frolov AA, Horak FB, Carlson-Kuhta P, Park S: Feedback equilibrium control during human standing. Biol Cybern 2005;93:309-322.

-10 Claudino R, dos Santos EC, Santos MJ: Compensatory but not anticipatory adjustments are altered in older adults during lateral postural perturbations. Clin Neurophysiol 2013;124:1628-1637.

11 Prieto TE, Myklebust JB, Hoffmann RG, Lovett EG, Myklebust BM: Measures of postural steadiness: differences between healthy young and elderly adults. IEEE Trans Biomed Eng 1996;43:956-966.

$>12$ Peterka RJ, Loughlin PJ: Dynamic regulation of sensorimotor integration in human postural control. J Neurophysiol 2004;91:410-423.

13 van Diest M, Lamoth CJ, Stegenga J, Verkerke GJ, Postema K: Exergaming for balance training of elderly: state of the art and future developments. J Neuroeng Rehabil 2013;10:101.

14 Carroll JP, Freedman W: Nonstationary properties of postural sway. J Biomech 1993;26:409-416.

15 Loughlin PJ, Redfern MS, Furman JM: Time-varying characteristics of visually induced postural sway. IEEE Trans Rehabil Eng 1996;4:416-424.

16 Newell KM, Slobounov SM, Slobounova E, Molenaar PMC: Short-term non-stationarity and the development of postural control. Gait Posture 1997;6:56-62.

17 Chong RK, Horak FB, Frank J, Kaye J: Sensory organization for balance: specific deficits in Alzheimer's but not in Parkinson's disease. J Gerontol A Biol Sci Med Sci 1999;54:M122-M128.

$\checkmark 18$ Maylor EA, Allison S, Wing AM: Effects of spatial and nonspatial cognitive activity on postural stability. Br J Psychol 2001;92:319-338.

19 Leandri M, Cammisuli S, Cammarata S, Baratto L, Campbell J, Simonini M, Tabaton M: Balance features in Alzheimer's disease and amnestic mild cognitive impairment. J Alzheimers Dis 2009;16:113-120.

-20 Yeh TT, Boulet J, Cluff T, Balasubramaniam R: Contributions of delayed visual feedback and cognitive task load to postural dynamics. Neurosci Lett 2010;481:173-177.

21 Yelshyna D, Gago MF, Bicho E, Fernandes V, Gago NF, Costa L, Silva H, Rodrigues ML, Rocha L, Sousa N: Compensatory postural adjustments in Parkinson's disease assessed via a virtual reality environment. Behav Brain Res 2016;296:384-392.

22 Schumann T, Redfern MS, Furman JM, el-Jaroudi A, Chaparro LF: Time-frequency analysis of postural sway. J Biomech 1995;28:603-607.

23 Boashash B: Time-frequency signal analysis; in Haykin SS (ed): Advances in Spectrum Analysis and Array Processing. Englewood Cliffs, Prentice-Hall, 1991, pp 418-517.

24 Papandreou-Suppappola A: Time-frequency processing: tutorial on principles and practice; in PapandreouSuppappola A (ed): Applications in Time-Frequency Signal Processing. Boca Raton, CRC, 2003, pp 1-84.

-25 Loughlin P, Pitton J, Hannaford B: Approximating time-frequency density functions via optimal combinations of spectrograms. IEEE Signal Process Lett 1994;1:199-202.

-26 Gago MF, Fernandes V, Ferreira J, Silva H, Rocha L, Bicho E, Sousa N: Postural stability analysis with inertial measurement units in Alzheimer's disease. Dement Geriatr Cogn Dis Extra 2014;4:22-30.

27 Stoffregen TA, Pagulayan RJ, Bardy BG, Hettinger LJ: Modulating postural control to facilitate visual performance. Hum Mov Sci 2000;19:203-220.

-28 Kenyon RV, Ellis SR: Vision, perception, and object manipulation in virtual environments; in Weiss PLT, Keshner EA, Levin MF (eds): Virtual Reality for Physical and Motor Rehabilitation. Virtual Reality Technologies for Health and Clinical Applications. New York, Springer, 2014, vol 1, pp 47-70.

29 Kobayashi K, Fushiki H, Asai M, Watanabe Y: Head and body sway in response to vertical visual stimulation. Acta Otolaryngol 2005;125:858-862.

-30 Dokka K, Kenyon RV, Keshner EA: Influence of visual scene velocity on segmental kinematics during stance. Gait Posture 2009;30:211-216.

-31 Riley MA, Clark S: Recurrence analysis of human postural sway during the sensory organization test. Neurosci Lett 2003;342:45-48.

-32 Martinez-Mendez R, Sekine M, Tamura T: Postural sway parameters using a triaxial accelerometer: comparing elderly and young healthy adults. Comput Methods Biomech Biomed Engin 2012;15:899-910.

33 Palmerini L, Rocchi L, Mellone S, Valzania F, Chiari L: Feature selection for accelerometer-based posture analysis in Parkinson's disease. IEEE Trans Inf Technol Biomed 2011;15:481-490.

-34 Watanabe T, Saito H, Koike E, Nitta K: A preliminary test of measurement of joint angles and stride length with wireless inertial sensors for wearable gait evaluation system. Comput Intell Neurosci 2011;2011:975193.

35 McKhann GM, Knopman DS, Chertkow H, Hyman BT, Jack CR Jr, Kawas CH, Klunk WE, Koroshetz WJ, Manly JJ, Mayeux R, Mohs RC, Morris JC, Rossor MN, Scheltens P, Carrillo MC, Thies B, Weintraub S, Phelps CH: The diagnosis of dementia due to Alzheimer's disease: recommendations from the National Institute on AgingAlzheimer's Association workgroups on diagnostic guidelines for Alzheimer's disease. Alzheimers Dement 2011;7:263-269.

-36 Lamb SE, Jørstad-Stein EC, Hauer K, Becker C; Prevention of Falls Network Europe and Outcomes Consensus Group: Development of a common outcome data set for fall injury prevention trials: the Prevention of Falls Network Europe consensus. J Am Geriatr Soc 2005;53:1618-1622.

-37 Freitas S, Simões MR, Alves L, Santana I: Montreal Cognitive Assessment (MoCA): normative study for the Portuguese population. J Clin Exp Neuropsychol 2011;33:989-996. 
-38 Afonso JA, Silva HD, Macedo P, Rocha LA: An enhanced reservation-based MAC protocol for IEEE 802.15.4 networks. Sensors (Basel) 2011;11:3852-3873.

39 Chong RK, Horak FB, Woollacott MH: Parkinson's disease impairs the ability to change set quickly. J Neurol Sci 2000;175:57-70.

40 Mathie MJ, Coster AC, Lovell NH, Celler BG: Accelerometry: providing an integrated, practical method for longterm, ambulatory monitoring of human movement. Physiol Meas 2004;25:R1-R20.

41 Williams ME: Geriatric Physical Diagnosis: A Guide to Observation and Assessment. Jefferson, McFarland \& Co, 2008.

42 Hunter MC, Hoffman MA: Postural control: visual and cognitive manipulations. Gait Posture 2001;13:41-48.

43 Loughlin PJ, Redfern MS: Spectral characteristics of visually induced postural sway in healthy elderly and healthy young subjects. IEEE Trans Neural Syst Rehabil Eng 2001;9:24-30.

-44 Ferdjallah M, Harris GF, Wertsch JJ: Instantaneous postural stability characterization using time-frequency analysis. Gait Posture 1999;10:129-134.

-45 van den Heuvel MR, Balasubramaniam R, Daffertshofer A, Longtin A, Beek PJ: Delayed visual feedback reveals distinct time scales in balance control. Neurosci Lett 2009;452:37-41.

46 Jordan MI, Wolpert DM: Computational motor control; in Gazzaniga SM (ed): The Cognitive Neurosciences, ed 2. Cambridge, MIT, 1999, pp 601-620.

47 Redfern MS, Jennings JR, Mendelson D, Nebes RD: Perceptual inhibition is associated with sensory integration in standing postural control among older adults. J Gerontol B Psychol Sci Soc Sci 2009;64:569-576.

-48 Horak FB: Postural orientation and equilibrium: what do we need to know about neural control of balance to prevent falls? Age Ageing 2006;35(suppl 2):ii7-ii11.

49 Gordon B, Carson K: The basis for choice reaction time slowing in Alzheimer's disease. Brain Cogn 1990;13: 148-166.

50 Lorbach ER, Webster KE, Menz HB, Wittwer JE, Merory JR: Physiological falls risk assessment in older people with Alzheimer's disease. Dement Geriatr Cogn Disord 2007;24:260-265.

51 Ehgoetz Martens KA, Ellard CG, Almeida QJ: Virtually-induced threat in Parkinson's: dopaminergic interactions between anxiety and sensory-perceptual processing while walking. Neuropsychologia 2015;79(pt B): 322-331.

52 Smith PF: Vestibular-hippocampal interactions. Hippocampus 1997;7:465-471.

53 Rizzo M, Nawrot M: Perception of movement and shape in Alzheimer's disease. Brain 1998;121:2259-2270.

54 Mendez MF, Cherrier MM, Meadows RS: Depth perception in Alzheimer's disease. Percept Mot Skills 1996;83: 987-995.

55 Cleworth TW, Chua R, Inglis JT, Carpenter MG: Influence of virtual height exposure on postural reactions to support surface translations. Gait Posture 2016;47:96-102.

56 Muir-Hunter SW, Clark J, McLean S, Pedlow S, Van Hemmen A, Montero Odasso M, Overend T: Identifying balance and fall risk in community-dwelling older women: the effect of executive function on postural control. Physiother Can 2014;66:179-186. 